\title{
Qualidades psicométricas do Inventário de Habilidades Sociais (IHS): estudo sobre a estabilidade temporal e a validade concomitante
}

\author{
Marina Bandeira \\ Monica Neves Costa \\ Fundação de Ensino Superior de São João del Rei \\ Zilda A. P. Del Prette \\ Almir Del Prette \\ Universidade Federal de São Carlos \\ Eliane Gerk-Carneiro \\ Universidade Gama Filho
}

Resumo

Esta pesquisa investiga as qualidades psicométricas do Inventário de Habilidades Sociais (IHS) em termos de sua validade concomitante e de sua fidedignidade ou estabilidade temporal. Participaram desta pesquisa, 104 estudantes de Psicologia que foram submetidos à aplicação do IHS e da Escala de Assertividade de Rathus. Os resultados mostram uma correlação significativa entre estas duas escalas de avaliação. Os dados referentes à aplicação teste-reteste do IHS, em uma subamostra aleatória de 39 sujeitos, mostram igualmente um correlação significativa entre as duas aplicações. Estes resultados indicam que o IHS possui validade concomitante e fidedignidade ou estabilidade temporal. Estas conclusões complementam os resultados de estudos anteriores sobre as qualidades psicométricas do IHS e recomendam a utilização desta escala para avaliar as habilidades sociais de estudantes universitários no contexto brasileiro.

Palavras-chave: Habilidades sociais, Universitários, Escala de avaliação, Propriedades. psicométricas 
Key words: Social skills, College students, Selfreport inventories, Psychometric properties.
Abstract

Psychometric qualities of a Social Skills Inventory (IHS): A study of its temporal stability and concomitant validity This research investigates the psychometric properties of the Social Skills Inventory (IHS) in terms of its concomitant validity and reliability. A sample of 104 psychology students participated in this research. Two scales were applied to the subjects, the IHS and the Rathus Assertiveness Scale. The results showed a significant correlation between the IHS and the Rathus Scale scores. The IHS was also reapplied to a randomized sub-sample of 39 students. The results of this test-retest application also showed a significant correlation between these scores. The results indicate that the IHS has concomitant validity and reliability or temporal stability. This study adds positive results to a previous study investigating some other psychometric properties of the IHS and recommends the use of this scale for the evaluation of college student social skills.

área de estudos e de aplicação das habilidades sociais está
se desenvolvendo cada vez mais nos últimos anos, no cená-
rio internacional, assim como em nosso contexto. A crescente complexidade das demandas sociais, tanto no nível pessoal quanto profissional, requer cada vez mais das pessoas habilidades sociais elaboradas. O desenvolvimento das habilidades sociais se mostra primordial na promoção de interações sociais bem sucedidas. Além disso, as dificuldades de habilidades sociais apresentadas pelas pessoas têm sido muitas vezes associadas à presença de diversos distúrbios psicossociais ou psicossomáticos (Lange \& Jakubowski, 1976; Lipp, Haythornthwaite \& Anderson, 1996) ou a um funcionamento social deficitário dos pacientes psiquiátricos na comunidade (Mueser, Wallace \& Liberman, 1995). Em conseqüência, o treinamento das habilidades sociais tem sido amplamente utilizado no tratamento dos pro- 
blemas psicossociais e na reabilitação psiquiátrica (Mueser et al., 1995). A importância das habilidades sociais vem despertando o interesse de especialistas nesta área e multiplicando as pesquisas sobre o tema (Del Prette \& Del Prette, 1999).

Dois conceitos principais emergem nesta área - habilidades sociais e competência social - que são às vezes empregados de forma indiferenciada, mas que têm sido igualmente destacados como dois conceitos distintos (Argyle, 1994; Hops, 1983; McFall, 1982; Schlundt \& McFall, 1985). Para fins específicos de avaliação, é importante distinguir estes dois conceitos, pois esta diferenciação permitirá o desenvolvimento de instrumentos de medida de maior especificidade.

O conceito de competência social envolve uma avaliação ou julgamento a respeito da adequação do comportamento de uma pessoa e do efeito que produz em uma determinada situação, enquanto que o conceito de habilidades sociais envolve mais o aspecto descritivo dos comportamentos verbais e não-verbais necessários à competência social (McFall, 1982; Del Prette \& Del Prette, 1999). Além disso, dentro do próprio conceito de habilidades sociais, inclui-se uma subárea que se refere à assertividade, a qual consiste na habilidade de afirmação e defesa dos próprios direitos, através da expressão de pensamentos, sentimentos e crenças, de forma direta e honesta, sem desrespeitar o direito dos outros (Lange \& Jakubowski, 1976).

$\mathrm{O}$ desenvolvimento da área das habilidades sociais cresceu juntamente com a necessidade de avaliação deste construto, que é definido como um desempenho e não como um traço. Enquanto desempenho, as habilidades sociais apresentam uma característica de especificidade situacional, o que pressupõe uma avaliação a partir de variadas dimensões situacionais e culturais. A avaliação das habilidades sociais requer a utilização de instrumentos de medida válidos e fidedignos, que sejam capazes de medir adequadamente estas habilidades, com o objetivo de adquirir conhecimento sobre o repertório dos sujeitos ou para avaliar os efeitos de programas de treinamento (Bellack, 1983; Lange \& Jakubowski, 1976).

Para a avaliação das habilidades sociais, foram desenvolvidos diversos tipos de instrumentos de medida, tais como o desempenho 
de papéis em situações planejadas, as medidas baseadas em entrevistas, a observação de interações naturais do sujeito com pessoas de seu ambiente e os instrumentos de auto-relato sob forma de escalas ou inventários de avaliação. Cada um destes tipos de avaliação apresenta vantagens e desvantagens particulares (Bandeira, 1999; Del Prette \& Del Prette, 1999). Dentro das categorias citadas acima, embora diversos instrumentos de medida já tenham sido construídos na área de estudo das habilidades sociais, poucos foram elaborados ou validados para o nosso contexto cultural. No caso particular da avaliação através do desempenho de papéis, raras são as escalas que foram elaboradas e validadas para o nosso contexto, tal como a escala elaborada por Bandeira e Tremblay (1998) visando uma população clínica particular de pacientes psiquiátricos. A escala elaborada por Lipp et al. (1996), contendo situações dirigidas para uma clientela mais ampla ainda não foi validada para o nosso contexto.

Um dos instrumentos de medida mais freqüentemente utilizados para avaliar as habilidades sociais, principalmente no caso de uma clientela não-clínica, consiste nos auto-relatos sob forma de questionários e inventários. Por ser de fácil aplicação, é considerado um instrumento ideal para ser utilizado em pesquisas de levantamentos amplos (surveys). Além disso, permite cobrir um grande número de situações e comportamentos e facilita a determinação de um padrão normativo em ampla escala. Entretanto, os inventários de avaliação das habilidades sociais, atualmente disponíveis na literatura desta área, também foram construídos em outros contextos culturais e temos informações limitadas acerca de suas qualidades psicométricas (Del Prette, Del Prette \& Barreto, 1998). Poucos são os inventários elaborados especificamente para o nosso contexto, tal como a Escala Brasileira para a Medida de Assertividade (Ayres, 1994) ou então escalas originárias de outra cultura adaptadas e validadas para o nosso contexto, tal como a Escala de Assertividade de Rathus (Pasquali \& Gouveia, 1990). Entretanto, estes dois instrumentos visam avaliar especificamente a assertividade e não a habilidade social, que consiste em um conceito mais abrangente (Del Prette, Del Prette \& Barreto, 1999). Considerando essa lacuna, tornou-se importante a elaboração 
de instrumentos avaliativos do desempenho interpessoal em nosso contexto.

Para suprir esta necessidade, Del Prette e Del Prette (2000) elaboraram o Inventário de Habilidades Sociais (IHS), para avaliar o repertório interpessoal de estudantes universitários. Em sua versão mais estudada do ponto de vista psicométrico, este inventário continha 38 itens. O IHS foi construído a partir do levantamento das situações que são mais pertinentes ao conceito de habilidades sociais e de pesquisas realizados pelos autores com universitários. Os itens deste instrumento de medida englobam variados contextos (público, privado ou indefinido), diversos tipos de interlocutores (familiar, desconhecido, autoridade, dentre outros) e variadas demandas interpessoais (reações a comportamentos desejáveis ou indesejáveis do interlocutor e emissões que não dependiam da ação explícita do interlocutor).

As propriedades psicométricas do IHS foram analisadas a partir das respostas de uma amostra de universitários de duas escolas de Uberlândia, sendo priorizada a análise da estrutura fatorial e da consistência interna do instrumento (Del Prette et al., 1998). Esse estudo identificou qualidades psicométricas adequadas do IHS. Os resultados mostraram que o inventário apresentou capacidade discriminativa adequada dos itens, uma consistência interna satisfatória (alpha de Cronbach $=0,75$ ) e uma estrutura multidimensional que incluía 5 fatores: enfrentamento com risco, auto-afirmação na expressão de afeto positivo, conversação e desenvoltura social, auto-exposição a desconhecidos ou a situações novas e autocontrole da agressividade em situações aversivas. Nesta estrutura, ficou evidenciada, pela porcentagem de variância explicada dos fatores, a importância do conceito de assertividade, que de fato consiste na subárea mais importante das habilidades sociais.

Outro estudo (Del Prette et al., 1999) sobre os efeitos de um programa de treinamento das habilidades sociais constatou ainda a sensibilidade do IHS em detectar modificações produzidas por este treinamento junto a um grupo de alunos do Curso de Psicologia da UFSCar comparativamente a um grupo controle da mesma classe. Os resultados mostraram que o IHS detectou um aumento das habilidades soci- 
ais no grupo submetido ao treinamento e ausência de efeito no grupo controle. Além disso, a constatação de ausência de mudanças no grupo controle, através da aplicação teste-reteste do IHS, serviu igualmente para atestar a estabilidade temporal deste inventário. Os resultados deste segundo estudo corroboram, portanto, os dados anteriores referentes à validade de construto e à fidedignidade do IHS.

Nos estudos de validação descritos acima, não foi realizada uma análise da validade concomitante do IHS, que constitui uma das análises necessárias para um conhecimento mais completo das qualidades psicométricas de um instrumento de medida. Esta análise permitiria verificar até que ponto os resultados obtidos através do IHS se assemelham, em uma mesma amostra de sujeito, aos observados por meio de um outro instrumento de medida (já validado) do mesmo construto ou um construto correlato. Além disso, no último estudo descrito, a análise da estabilidade temporal do IHS foi feita através de uma avaliação teste-reteste com uma amostra não-aleatória de alunos de psicologia, restrita a 23 componentes. Uma reavaliação desta fidedignidade através do teste-reteste em uma nova amostra aleatória seria desejável para consolidar este conhecimento sobre o IHS.

O objetivo do presente trabalho é, portanto, complementar as pesquisas descritas acima, no sentido de ampliar a análise das propriedades psicométricas do IHS, acrescentando um estudo de sua validade concomitante e reavaliando sua fidedignidade temporal, em uma nova amostra de alunos universitários. A validade concomitante do IHS será avaliada através de uma análise da correlação entre os resultados deste inventário e os da Escala de Assertividade de Rathus (Pasquali \& Gouveia, 1990), já validada anteriormente. O estudo da fidedignidade ou estabilidade temporal do IHS será realizado por meio de teste-reteste em uma amostra aleatória de sujeitos.

No presente estudo, será analisada a versão mais recente do IHS (Del Prette \& Del Prette, 2000, Apêndice A), que inclui a reformulação de algumas questões visando maior discriminabilidade das mesmas (Del Prette et al., 1998), assim como a inclusão de quatro novas questões que foram consideradas importantes para uma avaliação mais completa das habilidades sociais. Esta nova versão está sendo igualmente submetida a uma nova análise fatorial dos itens e a uma análise 
de sua consistência interna, através de um estudo multicêntrico em quatro regiões do país e em diferentes países da América Latina. (Del Prette et al., 2000).

\section{Método}

\section{Participantes}

A amostra da presente pesquisa consiste de 104 alunos do Curso de Psicologia da Fundação de Ensino Superior de São João del Rei (FUNREI), dos turnos integral e noturno, de ambos os sexos. A idade média dos estudantes é de 22,68 anos (D. P. = 4,36) e a maioria é do sexo feminino $(85,6 \%)$.

No que se refere ao tipo de ocupação dos estudantes, verificouse que a maioria se dedicava integralmente aos estudos, poucos trabalhavam $(26,9 \%)$ e somente uma minoria possuía bolsa de estudo $(2,9 \%)$.

\section{Instrumentos de Medida}

Para se verificar a validade concomitante do IHS, os participantes responderam aos dois instrumentos descritos abaixo:

Inventário de Habilidades Sociais (IHS - Apêndice A). Contém 42 itens, cada um apresentando uma ação ou sentimento diante de uma determinada situação social. Os alunos indicavam a freqüência com que agiam ou se sentiam da maneira como estava descrito em cada item. Para responder, era necessário preencher uma folha de respostas situada ao final dos itens, onde constava a legenda de uma escala tipo Likert, com cinco pontos: A (nunca ou raramente), B (com pouca freqüência), $\mathrm{C}$ (com regular freqüência), D (muito freqüentemente), $\mathrm{E}$ (sempre ou quase sempre). As informações referentes aos dados sócio-demográficos dos sujeitos eram preenchidas no cabeçalho da folha de respostas.

Escala de Assertividade de Rathus (Rathus, 1973): Este instrumento, validado para o Brasil (Pasquali \& Gouveia, 1990), possui 30 questões que visam avaliar o grau de assertividade das pessoas. Os itens descrevem comportamentos ou sentimentos que ocorrem em situações sociais cotidianas. Os participantes da pesquisa respondi- 
am às questões, utilizando um código com números que variavam de -3 a +3 , o que compreendia 6 tipos de respostas : +3 : extremamente parecido comigo; +2 : bastante parecido comigo; +1 : relativamente parecido comigo; -1: relativamente diferente de mim; -2 : bastante diferente de mim; -3: extremamente diferente de mim. Deste modo, as respostas indicam a intensidade da reação do sujeito diante das situações, ou seja, o quanto identifica as reações descritas com o seu modo de ser e de agir.

Esta escala apresenta um bom índice de estabilidade temporal ou fidedignidade, verificada através do método de correlação teste-reteste $(r=0,778)$ e do método de correlação das duas metades $(r=0,772)$. Por outro lado, sua validade concomitante foi estabelecida através de dois critérios. O primeiro critério foi um teste de diferencial semântico respondido por colegas dos sujeitos da pesquisa, sendo estes últimos avaliados em termos da impressão que causavam nas outras pessoas. O segundo critério foi uma avaliação da reação dos sujeitos a cinco situações sociais que exigiam comportamentos assertivos, tendo sido significativa a correlação desses escores com os da Escala de Rathus $(r=0,705)$.

\section{Procedimentos}

A aplicação do IHS e da Escala de Assertividade de Rathus ocorreu em grupo nas salas de aula dos alunos do curso de Psicologia. $\mathrm{O}$ aplicador seguia uma folha de instruções para aplicação dos instrumentos, para garantir a padronização deste procedimento. Ele explicava aos alunos os objetivos da pesquisa e apresentava as instruções sobre como responder, deixando claro que não havia respostas certas ou erradas e garantindo o anonimato do respondente. Além disso, o aplicador verificava se as instruções tinham sido compreendidas pelos estudantes e conferia se todos os itens haviam sido respondidos, a fim de diminuir a probabilidade de ocorrência de erros ou de questões em branco.

A fidedignidade do inventário IHS foi avaliada através do estudo da sua estabilidade temporal, usando-se o procedimento de testereteste. Após três semanas de aplicação do IHS, foi selecionada alea- 
toriamente uma sub-amostra de 39 estudantes, os quais foram submetidos a uma nova aplicação do IHS.

\section{Análise dos Dados}

Os dados da presente pesquisa, referentes à fidedignidade e à validade concomitante do IHS, foram submetidos a uma análise correlacional de Spearman, adequada para dados não-paramétricos, através do pacote estatístico Statistical Package for Social Science (SPSS-PC).

\section{Resultados}

\section{Qualidades Psicométricas do IHS}

Fidedignidade do IHS: A fidedignidade do IHS foi avaliada através de uma análise correlacional entre os escores das duas aplicações teste-reteste do IHS. A Tabela 1 apresenta as médias e desvios-padrão obtidos na sub-amostra de sujeitos submetidos a estas duas aplicações, assim como o valor do coeficiente de correlação obtido.

Tabela 1.

Médias e desvios-padrão dos escores do IHS no teste e reteste aplicados a uma sub-amostra dos sujeitos e coeficiente de correlação de Spearman entre os dois escores

\begin{tabular}{lcccc}
\hline $\begin{array}{c}\text { Aplicações } \\
\text { do IHS }\end{array}$ & $\mathrm{N}$ & $\begin{array}{c}\text { Desvios- } \\
\text { padrão }\end{array}$ & Médias & Teste-reteste \\
\hline IHS teste & 39 & 0,49 & 3,46 & \\
IHS reteste & 39 & 0,52 & 3,33 & $0,90^{* *}$ \\
\hline
\end{tabular}

$* * \mathrm{p}=0.001$

Os resultados mostram que houve uma correlação alta e significativa $(r=0,90, p=0,001)$ entre as duas aplicações do IHS, demonstrando a qualidade de fidedignidade ou estabilidade temporal deste inventário. Estes resultados indicam, portanto, que o IHS constitui um instrumento de medida confiável das habilidades sociais de estudantes universitários. 
Validade concomitante: A validade concomitante do IHS foi avaliada através de uma análise correlacional entre os dados deste inventário e os da Escala de Assertividade de Rathus. A Tabela 2 apresenta as médias e desvios-padrão dos escores obtidos através do IHS e da Escala de Rathus, assim como as correlações entre eles. Os resultados desta análise mostram uma correlação significativa entre as duas escalas $(r=0,79, p=0,01)$. Uma correlação mais elevada $(r=0,81, p=$ $0,01)$ foi obtida entre o IHS e os 20 itens da Escala de Rathus que compõem o principal fator desta escala e que constituem, segundo Pasquali e Gouveia (1990), um índice mais válido e fidedigno do construto avaliado, uma vez que foram os únicos itens que apresentaram cargas fatoriais iguais ou superiores a 0,30 . Tendo em vista este critério mínimo adotado, de fato apenas estes 20 itens da escala de Rathus deveriam ser aplicados no nosso contexto, pois os demais itens não alcançam a carga fatorial mínima necessária para uma validade de construto adequada e portanto devem ser eliminados da escala. Portanto, a correlação mais elevada do IHS com os 20 itens mais válidos da Escala de Rathus corrobora a validade do IHS.

Tabela 2.

Médias e desvios-padrão dos escores do IHS e da Escala de Rathus e correlação de Spearman entre os escores do IHS, do Rathus e dos 20 itens do primeiro fator da Escala de Rathus

\begin{tabular}{lcccc}
\hline \multicolumn{1}{c}{ Testes } & $\mathrm{N}$ & Média & $\begin{array}{c}\text { Desvios- } \\
\text { padrão }\end{array}$ & $\mathrm{R}$ \\
\hline IHS & 104 & 3,55 & 0,47 & \\
Rathus & 104 & 0,26 & 0,79 & $0,79 * *$ \\
Rathus-20 & 104 & 0,21 & 0,93 & $0,81^{* *}$ \\
\hline${ }^{* * \mathrm{p}=0.001}$ & & & &
\end{tabular}

Estes resultados mostram que os dados obtidos através do IHS são concordantes com aqueles obtidos através de uma escala que avalia um construto correlato, sendo esta última já validada para o nosso contexto, o que demonstra a validade concomitante do IHS. 


\section{Conclusões}

Os resultados da presente pesquisa complementaram as conclusões a respeito da adequação das qualidades psicométricas do IHS (Del Prette et al., 1998). Foi constatado no presente trabalho que este inventário de habilidades sociais apresenta validade concomitante, uma vez que as avaliações dos estudantes feitas através do IHS concordam com as obtidas através da Escala de Rathus, a qual mede um construto correlato e interdependente. Considerando que as duas escalas medem construtos correlatos, mas não idênticos, o valor da correlação obtida no presente trabalho encontra-se dentro da faixa esperada. Os resultados aqui obtidos servem para aumentar a segurança de que o IHS é um instrumento válido para medir o construto de habilidades sociais. Como no estudo anterior a análise das propriedades psicométricas do IHS não havia incluído uma análise da validade concomitante, a conclusão do presente trabalho contribui, portanto, para ampliar o conhecimento a respeito das propriedades psicométricas deste inventário.

A presente pesquisa demonstrou também a fidedignidade ou estabilidade temporal do IHS, já que a correlação obtida através do método do teste-reteste foi alta e significativa. Estes resultados confirmam os dados obtidos anteriormente (Del Prette et al., 1992), que haviam demonstrado a estabilidade temporal deste inventário em uma amostra menor e não-aleatória. Podemos concluir, portanto, que as avaliações efetuadas através deste inventário são confiáveis.

Os resultados aqui apresentados servirão para complementar a nova análise que está sendo feita atualmente sobre as propriedades psicométricas do IHS, em um estudo multicêntrico através de diferentes regiões do país, utilizando-se a versão mais recente do IHS, como a empregada no presente trabalho (Del Prette et al., 2000).

Tendo em vista os indicadores da validade e da confiabilidade do IHS, podemos concluir que este inventário possui as qualidades psicométricas necessárias para ser utilizado no contexto universitário brasileiro, visando objetivos clínicos ou de pesquisa que envolvam as habilidades sociais. Além de ser uma alternativa mais adequada do ponto de vista psicométrico, o IHS é de fácil aplicação, permitindo sua 
utilização em pesquisas de levantamento em larga escala. Este inventário permite avaliar as habilidades sociais a partir de variadas dimensões comportamentais e situacionais deste conceito e contempla situações pertinentes à cultura de nosso país. A possibilidade de acesso a um inventário deste tipo servirá para evitar as dificuldades metodológicas da utilização de instrumentos não padronizados para o contexto brasileiro.

\section{Referências}

Ayres, L. S. M. (1994). Uma escala brasileira para a medida da assertividade. Dissertação de mestrado não-publicada, Universidade Gama Filho, Rio de Janeiro.

Argyle, M. (1994). Psicologia del comportamiento interpersonal. Madrid: Alianza Universidad.

Bandeira, M. (1999). Competência social de psicóticos: parâmetros do treinamento para programas de reabilitação psiquiátrica - Parte II. Jornal Brasileiro de Psiquiatria, 48 (5), 191-195.

Bandeira, M., \& Tremblay, L. (1998). Competência social de psicóticos: um estudo de validação social. Jornal Brasileiro de Psiquiatria, 47 (4), 185-192.

Bellack, A. S. (1983). Recurrent problems in the behavioral assessment of social skill. Behavior Resarch and Therapy, 21 (1), 29-41.

Del Prette, Z. A. P., \& Del Prette, A. (1999). Psicologia das habilidades sociais: terapia e educação. Rio de Janeiro: Vozes.

Del Prette, A., \& Del Prette, Z. A. P. (2000). Inventário de Habilidades Sociais. Texto do inventário. Manuscrito não-publicado.

Del Prette, Z. A. P., Del Prette, A., \& Barreto, M. C. M. (1998). Análise de um Inventário de Habilidades Sociais (IHS) em uma amostra de universitários. Psicologia: Teoria e Pesquisa, 14 (3), 219-228.

Del Prette, A., Del Prette, Z. A. P., \& Barreto, M. C. M. (1999). Habilidades sociales en la formación del psicólogo: Análisis de un programa de intervención. Psicología Conductual, 7, 27-47.

Del Prette, A.; Del Prette, Z. A. P., \& Castelo Branco, U. V. (1992). Competência social na formação do psicólogo. Paidéia: Cadernos de Educação, 2, 4050 .

Del Prette, Z. A. P., Del Prette, A., Barreto, M. C. M, Caballo, V. E., RiosSaldaña, M. R., Oliveira, A. L. A. O., Falcone, E., Bandeira, M., \& GerkCarneiro, E. (2000). Habilidades sociais de estudantes de Psicologia: um estudo transcultural em diferentes regiões brasileiras e em diferentes países de língua latina. Projeto em andamento. 
Hops, H. (1983). Children's social competence and skill: Current research practices and future directions. Behavior Therapy, 14, 3-18.

Lange, J .L., \& Jakubowski, P. (1976). Responsible Assertive Behavior. Illinois: Research Press.

Lipp, M. N., Haythornthwaite, J., \& Anderson, D. E. (1996). Medidas diversas da assertividade em adultos. Estudos de Psicologia (Campinas), 13 (1), 1926.

McFall, R. P. (1982). A review and formulation of the concept of social skills. Behavioral Assessment, 4, 1-33.

Mueser, K. T., Wallace, C. J., \& Liberman, R. P. (1995). New developments in social skills training. Behaviour Change, 12 (1), 31-40.

Pasquali, L., \& Gouveia, V. V. (1990). Escala de assertividade de Rathus - RAS: adaptação brasileira. Psicologia: Teoria e Pesquisa, 6 (3), 233-249.

Rathus, S. A. (1973). A 30-item schedule for assessing assertive behavior. Behavior Therapy, 4, 398-406.

Schlundt, D.G., \& McFall, R. M. (1985). New directions in the assessment of social competence and social skills. In L. L'Abate \& M.A. Milan (Org.), Handbook of Social Skills Training and Research (pp.361-387). New York: Wiley and Sons.

Marina Bandeira, doutora em Psicologia pela Université de Montréal, Canadá, é professora da Fundação de Ensino Superior de São João Del Rei (FUNREI), São João Del Rei, MG. Monica Neves Costa é aluna do Curso de Graduação em Psicologia da FUNREI. Zilda A. P. Del Prette, doutora em Psicologia Experimental pela Universidade de São Paulo, é professora do Departamento de Psicologia da Universidade Federal de São Carlos, SP. Almir Del Prette, doutor em Psicologia Experimental pela Universidade de São Paulo, é
Sobre os autores 
414 M.Bandeira et al.

professor do Departamento de Psicologia da Universidade Federal de São Carlos, SP. Eliane Gerk-Carnei$r o$, doutora em Processos Cognitivos pela Fundação Getúlio Vargas, é professora do Departamento de Psicologia da Universidade Gama Filho, Rio de Janeiro, RJ.

Endereço para correspondência: Marina Bandeira, Departamento de Psicologia, FUNREI, Praça Dom Helvecio, 74, 36.300-000, São João Del Rei, MG.

E-mails:

MB, marina@mgconecta.com.br; ZAPDP: zdprette@ power.ufscar.br; ADP: adprette@ power.ufscar.br; EG-C: gerk@hexanet.com.br. 


\section{Apêndice A \\ INVENTÁRIO DE HABILIDADES SOCIAIS}

Almir Del Prette e Zilda Aparecida Pereira Del Prette

Leia atentamente cada um dos ítens que se seguem. Cada um deles apresenta uma ação ou sentimento (parte grifada) diante de uma situação dada (parte não grifada). Indique, na FOLHA DE RESPOSTAS, a frequiência com que você age ou se sente tal como descrito no ítem. Se uma dessas situações nunca lhe ocorreu, responda como se tivesse ocorrido, considerando o seu possível comportamento. RESPONDA TODAS AS QUESTÕES.

1. Em um grupo de pessoas desconhecidas, fico à vontade, conversando naturalmente.

2. Quando um de meus familiares (pais, irmãos mais velhos ou cônjuge) insiste em dizer o que eu devo fazer, contrariando o que penso, acabo aceitando para evitar problemas.

3. Ao ser elogiado(a) sinceramente por alguém, respondo-lhe agradecendo

4. Em uma conversação, se uma pessoa me interrompe, solicito que aguarde até eu encerrar o que estava dizendo.

5. Quando um(a) amigo(a) a quem emprestei dinheiro, esquece de me devolver, encontro um jeito de lembrá-lo(a).

6. Quando alguém faz algo que eu acho bom, mesmo que não seja diretamente a mim, faço menção a isso, elogiando-o(a) na primeira oportunidade.

7. Ao sentir desejo de conhecer alguém a quem não fui apresentado(a), eu mesmo(a) me apresento a essa pessoa.

8. Mesmo junto a conhecidos da escola ou trabalho, encontro dificuldade em participar da conversação ("enturmar").

9. Evito fazer exposições ou palestras a pessoas desconhecidas.

10. Em minha casa expresso sentimentos de carinho através de palavras e gestos a meus familiares.

11. Em uma sala de aula ou reunião, se o professor ou dirigente faz uma afirmação incorreta, eu exponho meu ponto de vista. 
416 M.Bandeira et al.

12. Se estou interessado(a) em uma pessoa para relacionamento sexual, consigo abordá-la para iniciar conversação.

13. Em meu trabalho ou em minha escola, se alguém me faz um elogio, fico encabulado(a) sem saber o que dizer.

14. Faço exposição (por exemplo palestras) em sala de aula ou no trabalho, quando sou indicado(a).

15. Quando um familiar me critica injustamente, expresso meu aborrecimento diretamente a ele.

16. Em um grupo de pessoas conhecidas, se não concordo com a maioria, expresso verbalmente minha discordância.

17. Em uma conversação com amigos, tenho dificuldade em encerrar a minha participação, preferindo aguardar que outros o façam.

18. Quando um de meus familiares, por algum motivo, me critica, reajo de forma agressiva.

19. Mesmo encontrando-me próximo(a) de uma pessoa importante, a quem gostaria de conhecer, tenho dificuldade em abordá-la para iniciar conversação.

20. Quando estou gostando de alguém com quem venho saindo, tomo a iniciativa de expressar-lhe meus sentimentos.

21. Ao receber uma mercadoria com defeito, dirijo-me até a loja onde a comprei, exigindo a sua substituição.

22. Ao ser solicitado(a) por um(a) colega para colocar seu nome em um trabalho feito sem a sua participação, acabo aceitando mesmo achando que não devia.

23. Evito fazer perguntas a pessoas desconhecidas.

24. Tenho dificuldade em interromper uma conversa ao telefone mesmo com pessoas conhecidas.

25. Ao deixar de gostar de uma pessoa com quem vinha saindo, tenho dificuldade em romper o relacionamento.

26. Em campanhas de solidariedade, evito tarefas que envolvam pedir donativos ou favores a pessoas desconhecidas.

27. Se um(a) amigo(a) abusa de minha boa vontade, expresso-lhe diretamente meu desagrado.

28. Quando um de meus familiares (filhos, pais, irmãos, cônjuge) consegue alguma coisa importante pela qual se empenhou muito, eu o elogio pelo 
sеu sucesso.

29. Na escola ou no trabalho, quando não compreendo uma explicação sobre algo que estou interessado(a), faço as perguntas que julgo necessárias aо теи esclarecimento.

30. Em uma situação de grupo, quando alguém é injustiçado, reajo em sua defesa.

31. Ao entrar em um ambiente onde estão várias pessoas desconhecidas, cumprimento-as.

32. Ao sentir que preciso de ajuda, tenho facilidade em pedi-la a alguém de meu círculo de amizades.

33. Quando meu(minha) parceiro(a) insiste em fazer sexo sem o uso da camisinha, concordo para evitar que ele(a) fique irritado(a) ou magoado $(a)$.

34. No trabalho ou na escola, concordo em fazer as tarefas que me pedem e que não são da minha obrigação, mesmo sentindo um certo abuso nesses pedidos.

35. Se estou sentindo-me bem (feliz), expresso isso para as pessoas de meu círculo de amizades.

36. Quando estou com uma pessoa que acabei de conhecer, sinto dificuldade em manter um papo interessante.

37. Se preciso pedir um favor a um(a) colega, acabo desistindo de fazê-lo.

38. Consigo "levar na esportiva" as gozações de colegas de escola ou de trabalho a meu respeito.

39. Ao receber uma avaliação muito abaixo do que merecia, fico chateado(a) mas evito discuti-la com o professor.

40. Em uma situação de conflito de opiniões, consigo convencer os demais para a minha posição.

41. Em uma fila (banco, cinema, etc), se um estranho passa à minha frente, fico calado(a) sem manifestar meu desagrado.

42. Prefiro ocultar minha opinião a ferir sentimentos alheios, mesmo quando solicitado(a) a dizer o que penso. 


\section{M.Bandeira et al.}

\section{FOLHA DE RESPOSTAS}

\section{PREENCHA INICIALMENTE O CABEÇALHO ABAIXO}

Curso:

Idade: .......

Sexo: M( ) F( )

Data

№ de créditos concluídos até hoje: № de semestres já cursado

(inclua de outras universidades, caso seja transferido)

Trabalha? Sim ( ) Não ( )

Crédito Educativo: Sim ( ) Não ( )

Bolsa: Sim ( ) Não ( )

Caso seja bolsista, especifique o tipo:

( ) Iniciação Científica

( ) Bolsa

Atividade

( ) Bolsa Treinamento

( ) Bolsa Alimentação ( ) Outra

(Especifique)

Formação Do Pai:

Formação Da Mãe:
Alfabetizado ( ) 1o. Grau ( ) 20.Grau ( ) 3o. Grau ( )

Alfabetizada ( ) 1o. Grau ( ) 2o. Grau ( ) 3o. Grau ( )

2. RESPONDA AOS ITENS DO INVENTÁRIO NOS QUADROS ABAIXO:

Em cada um dos itens abaixo, faça um $\mathrm{X}$ no quadrinho que melhor indica a freqüência com que você apresenta a reação sugerida em cada item, considerando um total de 10 vezes em que poderia se encontrar na situação descrita no item. Utilize a seguinte legenda.

A - NUNCA OU RARAMENTE (em cada 10 situações desse tipo, reajo dessa forma no máximo 2 vezes)

B - COM POUCA FREQÜÊNCIA (em cada 10 situações desse tipo, reajo dessa forma 3 a 4 vezes)

C - COM REGULAR FREQÜÊNCIA (em cada 10 situações desse tipo, reajo dessa forma 4 a 6 vezes)

D - MUITO FREQUENTEMENTE (em cada 10 situações desse tipo, reajo dessa forma 6 a 8 vezes)

E - SEMPRE OU QUASE SEMPRE (em cada 10 situações desse tipo, reajo dessa forma 8 a 10 vezes) 
Estabilidade temporal e validade concomitante no IHS $\mathbf{4 1 9}$
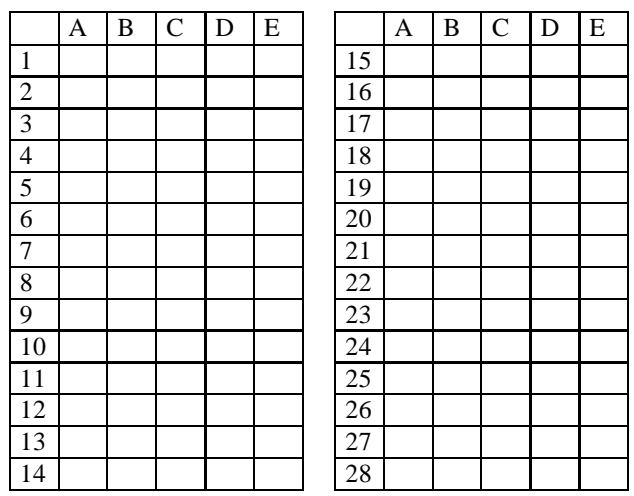

\begin{tabular}{|l|l|l|l|l|l|}
\hline & A & B & C & D & E \\
\hline 29 & & & & & \\
\hline 30 & & & & & \\
\hline 31 & & & & & \\
\hline 32 & & & & & \\
\hline 33 & & & & & \\
\hline 34 & & & & & \\
\hline 35 & & & & & \\
\hline 36 & & & & & \\
\hline 37 & & & & & \\
\hline 38 & & & & & \\
\hline 39 & & & & & \\
\hline 40 & & & & & \\
\hline 41 & & & & & \\
\hline 42 & & & & & \\
\hline
\end{tabular}

